\title{
VLBI Observations of Earth Orientation
}

C. $\mathrm{Ma}$

\author{
NASA Goddard Space Flight Center, Greenbelt, MD 20771
}

D. S. MacMillan

NVI, Incorporated and NASA Goddard Space Flight Center, Greenbelt, $M D 20771$

\begin{abstract}
VLBI has been used for high precision EOP measurements since 1980. The typical formal error for current routine monitoring of polar motion is $\sim 80 \mu$ as. A program for continuous EOP observations using VLBI is being incrementally implemented. There are unexplained differences between EOP values derived from simultaneously observing networks.
\end{abstract}

\section{Introduction}

VLBI (very long baseline interferometry) is the space geodetic technique that is uniquely capable of measuring Earth orientation parameters (EOP) because of its direct access to both the celestial and terrestrial reference frames. The VLBI celestial reference frame (CRF) in which Earth orientation is defined consists of several hundred extragalactic radio sources whose cosmological distances assure an overall kinematic stability of a small fraction of a milliarcsecond ( $\mathrm{Ma}$ et al. 1998). The VLBI terrestrial reference frame (TRF) consists of several tens of large radio antennas distributed on all major tectonic plates. Since VLBI is a purely geometric, ground-based technique, it is not complicated by the need to model satellite dynamics and the gravity field. However, its full potential for the highest resolution of EOP measurements has been limited by the cost of observing and data processing. These limitations will be overcome in large part by the introduction of Mark IV data acquisition terminals and correlators.

\section{Basic Principles of VLBI}

The fundamental measurement from VLBI is related to the length of the baseline between two antennas and the orientation of the baseline with respect to the observed radio source. Consequently, both linear measurements between terrestrial points and angular measurements between celestial points are relative. VLBI has no direct sensitivity to the origin of the terrestrial reference frame, unlike dynamical techniques like SLR or GPS. However, VLBI is sensitive to all components of Earth orientation, which is conventionally over-specified by two components of polar motion, UT1, and two components of nutation ( $\Delta \epsilon$ and 
$\Delta \psi$, offsets in obliquity and longitude from the standard models of nutation and precession). More precisely, VLBI is sensitive to differences in Earth orientation between observing epochs. Some initial set of EOP or CRF/TRF is needed. Starting from the initial conditions, the 20-year VLBI data set can be used to simultaneously estimate corrections to the TRF and CRF as well as to derive a consistent set of EOP. Because of the inherent stability of the VLBI CRF, the long-term stability of the EOP time series is limited only by the consistency and accuracy of the station positions and velocities.

\section{VLBI Observing Programs for Monitoring Earth Orientation}

The regular measurement of Earth orientation parameters by VLBI, including polar motion, developed in steps, advancing both with technology and coordination/funding of observing programs. The first proof of concept occurred during the short MERIT (Monitoring Earth Rotation and Intercomparison of Techniques) campaign in 1980. A network of stations in North America and Europe observed every day for two separate weeks. The clear success of this effort led to the beginning of regular monitoring of EOP with the two-station POLARIS network between Texas and Massachusetts undertaken by the U.S. National Geodetic Survey (NGS). With only one baseline, the measurements provided only one component of polar motion (X-pole) along with UT1. When new, dedicated geodetic VLBI stations were built in 1983 in southeastern U.S. and southern Germany, all EOP components became available through IRIS (International Radio Interferometric Surveying), variously undertaken by U.S., German and Japanese organizations. To address its specific requirements, the U.S. Naval Observatory (USNO) developed NavNet in 1989 using only U.S. stations. The two U.S. observing programs were combined in 1993 under NEOS (National Earth Orientation Service), a joint effort of USNO, NOAA and NASA with the collaboration of German, Brazilian, Norwegian and Canadian observatories. The CORE (Continuous Observations of the Rotation of the Earth) program to provide significantly enhanced resolution and precision in support of Earth system science was begun in 1997. It now observes with two types of networks, designated CORE-A and CORE-B. Table 1 is a summmary of the observing programs and the major networks are shown in Figures 1 and 2. There are 39 stations currently active.

\section{VLBI Reference Frames}

Because of the geometry of VLBI observations, the VLBI delay observable is invariant to translations of station positions and translations of station velocities. It is also invariant under two rotational transformations:

rotation of the TRF + EOP change = rotation of $\mathrm{CRF}$

rotation rate of $T R F=-E O P$ rate change

(The rotation rate of the CRF is assumed to be zero and source position rates are usually not estimated.) Since the TRF and CRF are normally adjusted to provide the best estimates, it is necessary to apply conditions to attach the VLBI TRF and CRF to a priori models so that the EOP reflect the relative orientation of the TRF and CRF. A usable set of conditions is the following. 
Table 1. EOP monitoring programs.

\begin{tabular}{ccccl}
\hline Program & Active & Frequency & $\begin{array}{c}\text { Precision } \\
\mu \text { as }\end{array}$ & Organizer \\
\hline MERIT & 1980 & daily & 600 & NASA, NGS \\
POLARIS & $1980-83$ & weekly & 3000 & NGS \\
IRIS-A & $1984-91$ & $5-$ day & $700-200$ & NGS \\
& $1991-93$ & weekly & 150 & NGS \\
IRIS-S & $1986-$ & monthly & $300-100$ & U. Bonn \\
IRIS-P & $1987-94$ & monthly & $400-200$ & IPMS Mizusawa \\
NavNet & $1989-93$ & weekly & $800-200$ & USNO \\
NEOS & $1993-$ & weekly & 80 & USNO, NASA, NOAA \\
CORE & $1997-$ & weekly & 80 & NASA \\
\hline vitimately daily & & & &
\end{tabular}

\section{POLARIS, IRIS and NavNet Networks}

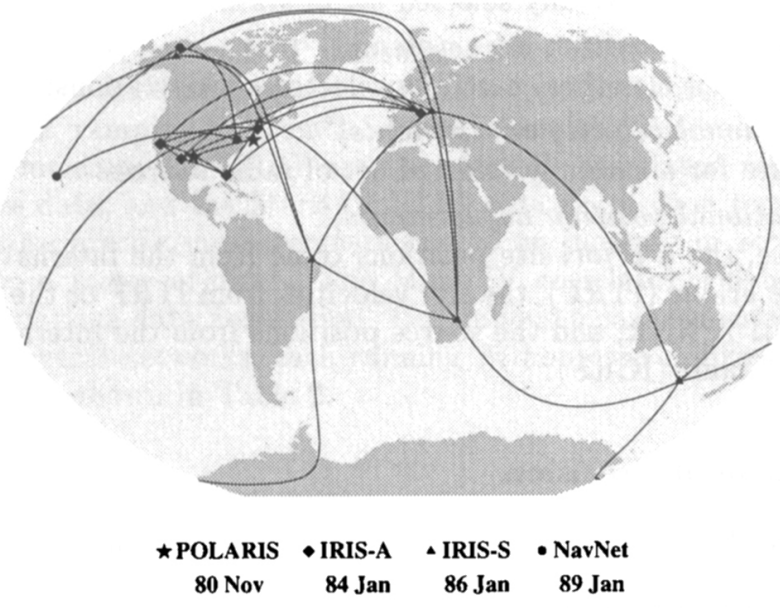

Figure 1. POLARIS, IRIS and NavNet networks. 


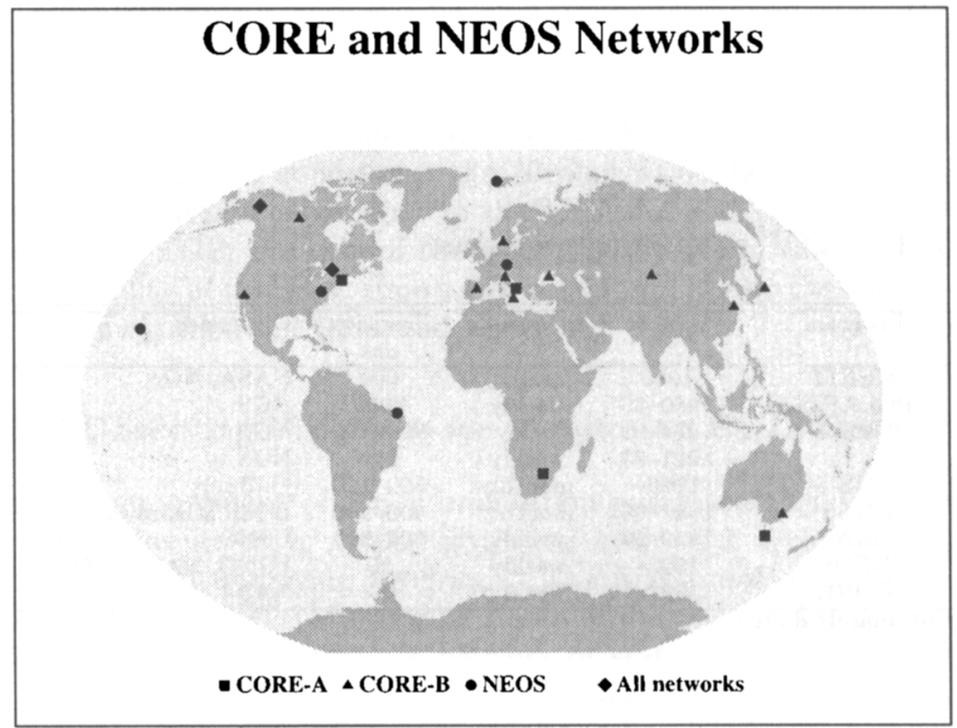

Figure 2. Stations of CORE and NEOS networks.

a) Minimize for a carefully selected set of stations:

$\sum \mid$ horizontal position adjustments $\left.\right|^{2}$

b) Minimize for a(nother) carefully selected set of stations:

$\sum \mid$ horizontal velocity adjustments $\left.\right|^{2}$

c) Minimize for a carefully selected set of stations:

$\sum \mid$ rotational position adjustments $\left.\right|^{2}$

d) Minimize for a(nother) carefully selected set of stations:

$\sum \mid$ rotational velocity adjustments $\left.\right|^{2}$

e) Minimize for a carefully selected set of radio sources:

$\sum \mid$ rotational position adjustments $\left.\right|^{2}$

In practice, the $a$ priori site positions come from the International Terrestrial Reference Frame (ITRF), the site velocities from ITRF or the plate motion model NUVEL1-A NNR, and the source positions from the International Celestial Reference Frame (ICRF).

\section{Progression of Precision}

The precision of VLBI measurements of polar motion has improved greatly over the past 20 years. Figure 3 shows the evolution of the precision of the $\mathrm{X}$ pole measurement. Figure 4 shows the corresponding information for Y-pole. The best VLBI measurements of polar motion have a precision of 30-40 $\mu$ as. It should be noted that these results come from a uniform analysis of the whole data set. The improvements therefore reflect changes in instrumentation, observing networks, sources, observing schedules (especially number of sources, number of observations per unit time and distribution of observations across the sky), and modeling. Similar figures made in the past with the same data then available 


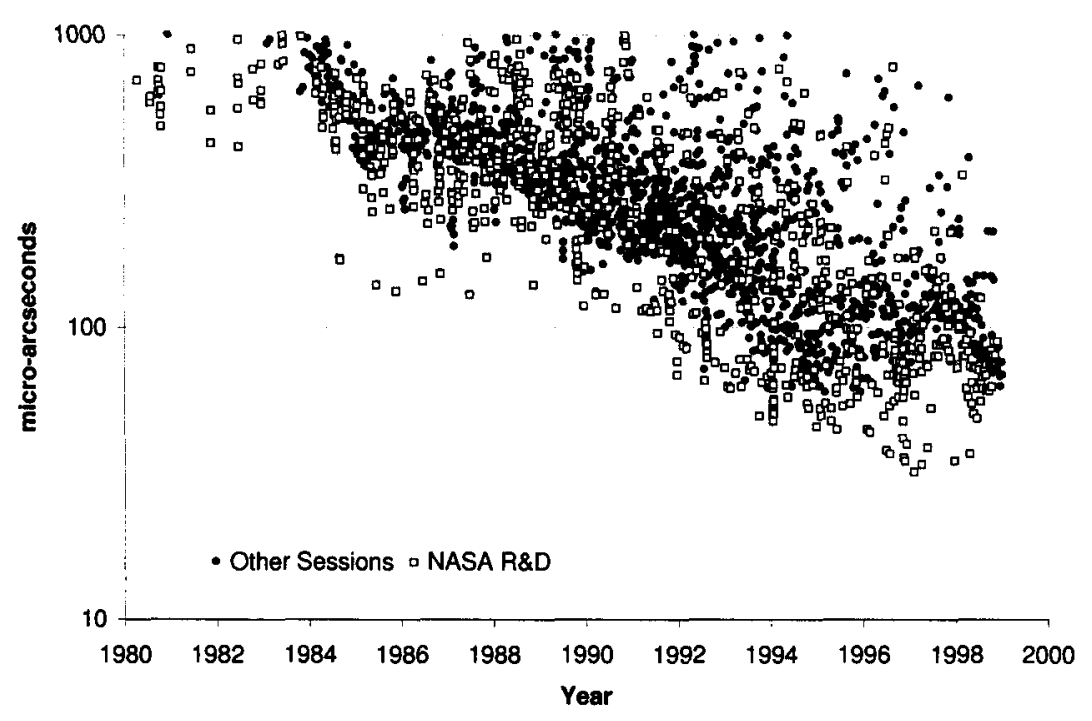

Figure 3. Formal errors of X-pole.

would show all errors larger because of less sophisticated analysis and more poorly determined TRF and CRF.

\section{CORE Measurements}

Recognizing the importance of EOP measurements, the international VLBI community is engaged in the CORE program to make continuous EOP measurements beginning in 2002, ramping up from 1.5 24-hour sessions per week during 1999. Two critical elements of the CORE are the Mark IV correlator, which can process much more data, and the Mark IV VLBI data acquisition terminal, which will provide more precise measurements due to an increase in recorded bandwidth by up to a factor of eight. The Mark IV correlator will be capable of supporting continuous data acquisition. Continuous measurements of EOP will be made with several networks, each running 24 hours on a different day. The accuracy goals are shown in Table 2.

Table 2. CORE accuracy goals.

\begin{tabular}{lccc}
\hline \hline & $1997-99$ & $2000-2001$ & $2002 \ldots$ \\
\hline UT1 & $3.5 \mu \mathrm{s}$ & $1.5 \mu \mathrm{s}$ & $1 \mu \mathrm{s}$ \\
X,Y pole & $100 \mu \mathrm{as}$ & $50 \mu \mathrm{as}$ & $25 \mu \mathrm{as}$ \\
Coverage & $1.5 \mathrm{~d} / \mathrm{wk}$ & $3.5 \mathrm{~d} / \mathrm{wk}$ & $5-7 \mathrm{~d} / \mathrm{wk}$ \\
\hline
\end{tabular}

One of the principal problems with high precision VLBI EOP is determining the measurement accuracy. In the CORE program, since EOP measurements will be made with several different networks, each network operating on a dif- 


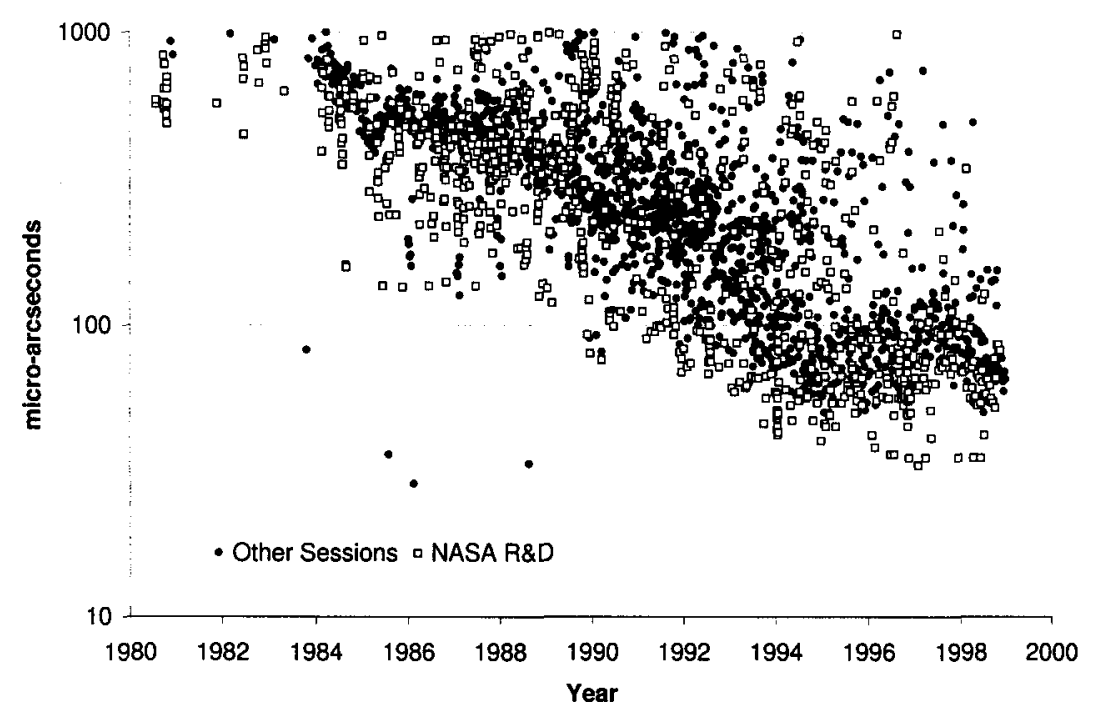

Figure 4. Formal errors of Y-pole.

ferent day, it is important to determine whether there are systematic differences between EOP derived from different networks. Our basic test of accuracy consists of examining the differences between EOP derived from independent VLBI measurements made by different networks.

From the set of VLBI sessions from 1980 to the present, there are about 300 simultaneous sessions. Comparisons of these simultaneous sessions imply that the formal EOP errors should be increased by a factor of 1.5-2.0. As can be seen in Figures 3 and 4, the precision of these measurements varies over a wide range, which is greater than a factor of ten compared to current precision. This reflects the evolution of the improvement of precision. Therefore it is difficult to determine from this comparison whether the differences are caused by random or systematic errors.

To determine the current EOP accuracy, we have compared measurements made by CORE and NEOS networks. The least-squares estimation program (SOLVE) used in the analysis and the theoretical models applied in the analysis are generally described by Ma et al. (1990). The weekly NEOS network uses Kokee, Green Bank, Fortaleza, Ny Ålesund, and Wettzell. The CORE-A network sessions are simultaneous with NEOS sessions and use antennas at Fairbanks, Westford, Algonquin Park, Hobart, Hartebeesthoek, and Matera. The networks are shown in Figure 5.

Figure 6 shows the differences between EOP measurements from the COREA and NEOS networks during 1997-1999. Based on the $\chi^{2} /$ deg of freedom of the differencess, which range from 1.3-2.0, there are clearly unmodeled sources of random or systematic error. Over this time period the actually observing networks have varied from the nominal set of stations because of unavailability of sites for certain observing sessions. A large component of the EOP differences shown in Figure 6 is due to changes in networks. 


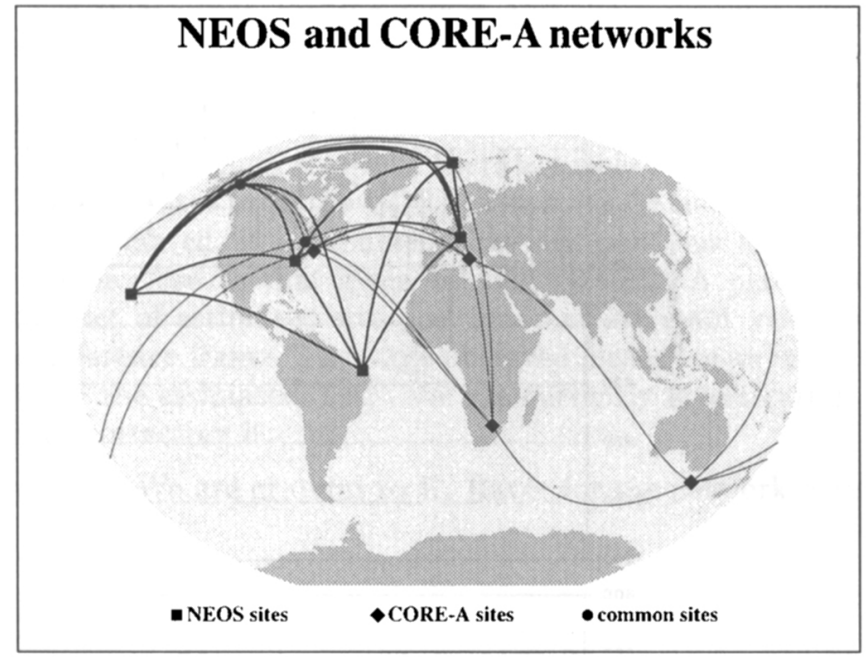

Figure 5. NEOS and CORE-A networks.

Analysis of the differences between NEOS and CORE-A EOP estimates for sessions in which each of the NEOS and CORE-A networks included exactly the nominal set of stations is summarized in Table 3.

Table 3. NEOS-CORE-A differences with consistent networks

\begin{tabular}{crrrr}
\hline \hline Component & $\begin{array}{r}\text { Bias } \\
\mu \text { as }\end{array}$ & $\chi^{2} /$ dof & $\begin{array}{r}\text { Wrms } \\
\mu \text { as }\end{array}$ & $\begin{array}{r}\langle\sigma\rangle \\
\mu \text { as }\end{array}$ \\
\hline X-Pole & $-133 \pm 37$ & 1.1 & 152 & 146 \\
Y-Pole & $-57 \pm 31$ & 1.1 & 126 & 123 \\
UT1 & $127 \pm 23$ & 1.9 & 122 & 90 \\
\hline
\end{tabular}

After removing the biases, the residual rms scatter for each difference pair is 100-150 $\mu$ as. The observed rms scatter is somewhat greater than the formal uncertainty in each case. The $\chi^{2} / \mathrm{deg}$ of freedom indicates that the formal uncertainties are too small by factors ranging from 1-1.4, where the largest factor is for the UT1 differences. We can estimate the size of the effect of unmodeled station motion error by simulating the effect of a 1-mm station displacement at each of the sites in the CORE-A and NEOS networks. One result is that horizontal displacements couple much more strongly to EOP estimates than vertical displacements (by a factor of 5-10). This is fortunate in the sense that most geophysical effects result predominantly in vertical change. Assuming that unmodeled errors are random, simulations show that the effect of $1-\mathrm{mm} \mathrm{rms}$ changes in the horizontal coordinates of all stations in the CORE-A network result in X-pole, Y-pole, and UT1 rms errors of 30-40 $\mu$ as. There are several possible sources of unmodeled error that could contribute to observed residual station position motion. Errors in tropospheric gradient modeling are probably not more than $20 \%$ of the effect of the current gradient model or about 15- 

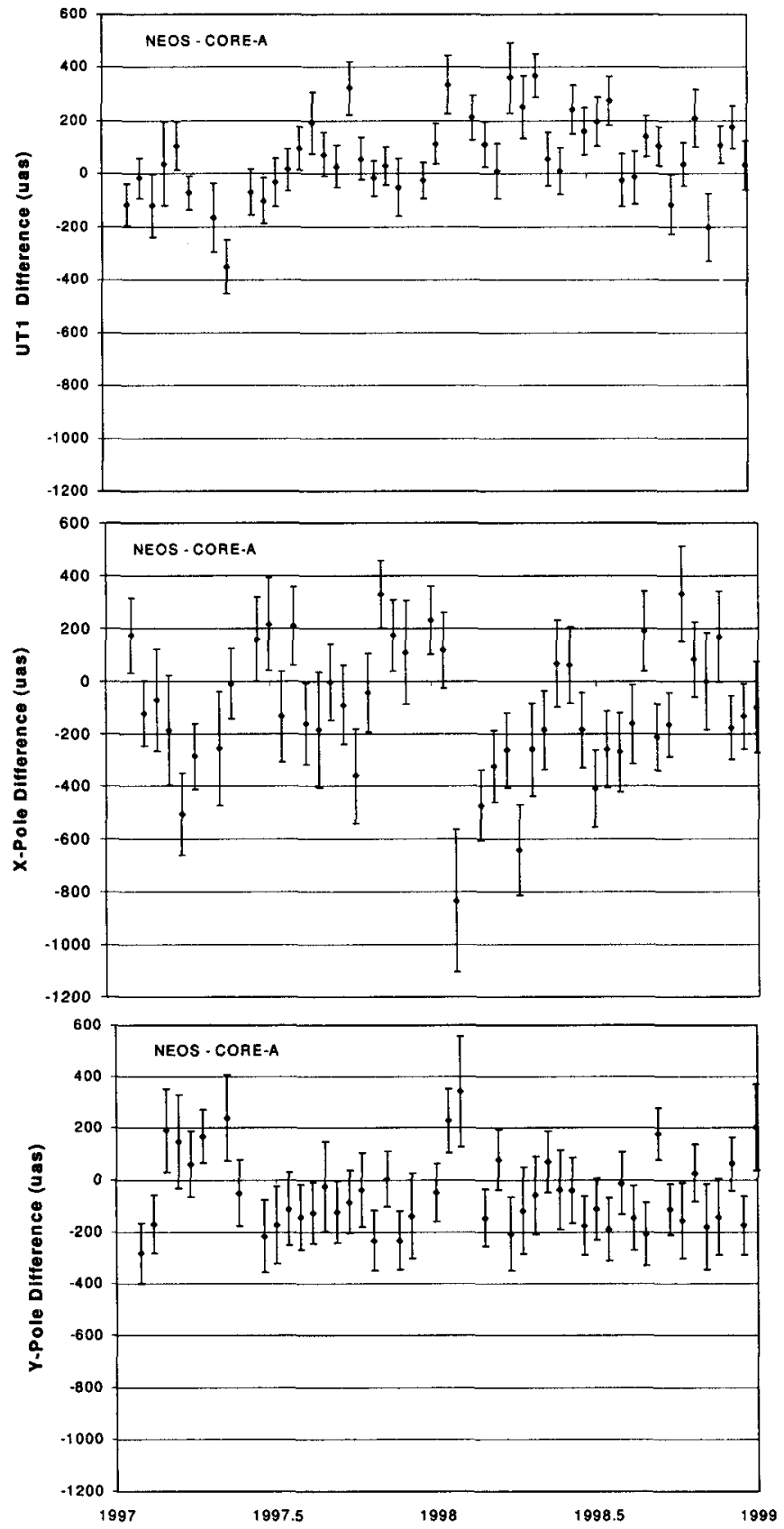

Figure 6. EOP differences: NEOS minus CORE-A. 
$30 \mu$ as. Errors in the tidal ocean loading model and the horizontal effect of atmospheric loading are each expected to contribute 10-40 $\mu$ as of rms variation to each EOP component. Unmodeled tropospheric and loading effects would then contribute 30-50 $\mu$ as of EOP error.

The comparison in Table 3 indicates that there are significant biases of 60 $130 \mu$ as between EOP estimates from the CORE-A and NEOS networks. When EOP parameters are estimated, it is assumed that site positions are evolving linearly at a rate that is close to that given by the NUVEL1-A plate motion model. The resulting set of estimated site positions and constant velocities is the VLBI terrestrial reference frame. The large observed biases are an indication that there are errors in the estimated TRF. We are currently investigating this error and methods for correcting it.

Acknowledgments. We are grateful to $\mathrm{K}$. Baver for the network maps.

\section{References}

Ma, C., Sauber, J. M., Bell, L. J., Clark, T. A., Gordon, D., Himwich, W. E., \& Ryan, J. R. 1990, J. Geophys. Res., 95, 21991.

Ma, C., Arias, E. F., Eubanks, T. M., Fey, A. L., Gontier, A.-M., Jacobs, C. S., Sovers, O. J., Archinal, B. A., \& Charlot, P. 1998, AJ, 116, 516. 


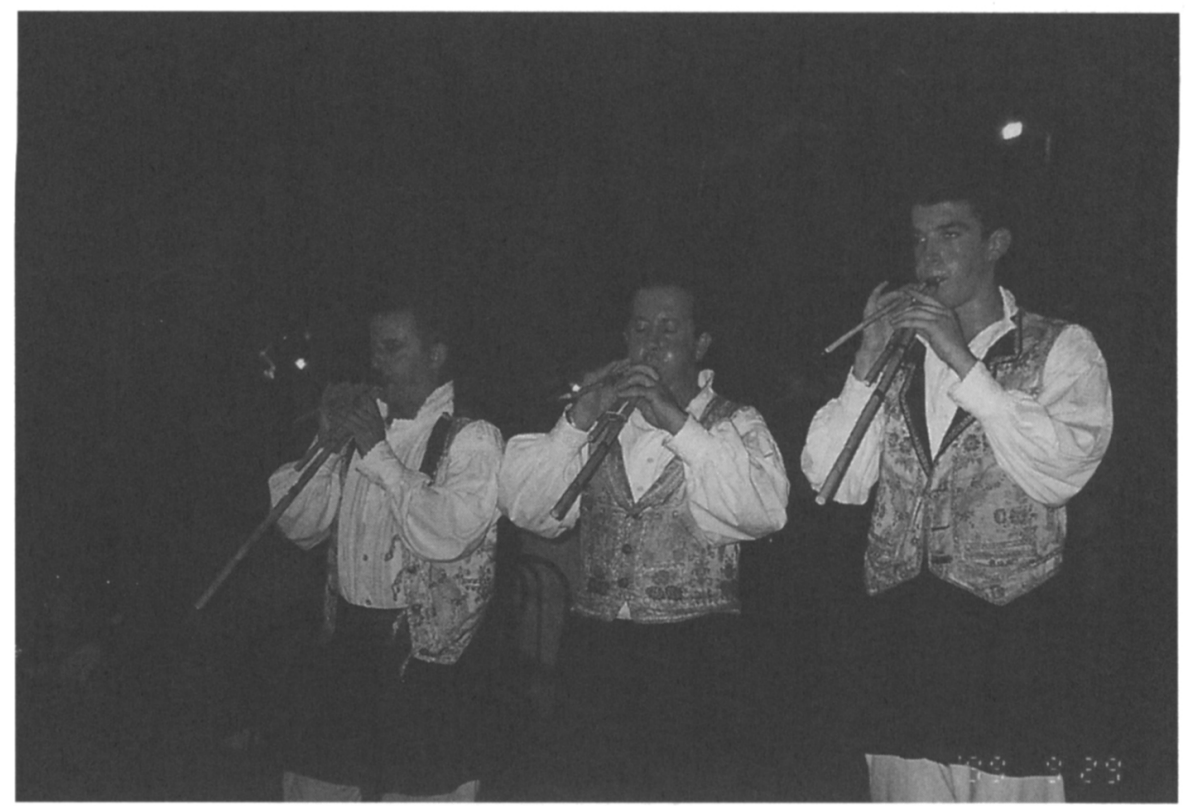

Traditional Sardinian Entertainment at the Banquet 